\title{
Research on the Evaluation of Cross-regional Environmental Collaborative Governance Ability -Take the Beijing-Tianjin-Hebei Urban Agglomeration as an Example
}

\author{
Moyang Cui ${ }^{1}$, Yunpeng Yang², Shuai Liu ${ }^{3}$, Yunhan Liu ${ }^{4}$ \\ ${ }^{1}$ Glorious Sun School of Business and Management, DongHua University, Shanghai, China \\ ${ }^{2}$ Antai College of Economics and Management, Shanghai Jiao Tong University, Shanghai, China \\ ${ }^{3}$ The College of Information, Mechanical and Electrical Engineering, Shanghai Normal University, Shanghai, China \\ ${ }^{4}$ University of Portsmouth, England, United Kingdom
}

\begin{abstract}
The cross-regional environmental collaborative governance capability evaluation problem is a heterogeneous multi-attribute decision-making problem. In response to this problem, this paper first constructed a cross-regional environmental collaborative governance capability evaluation index system, including five dimensions, involving 25 specific indicators with heterogeneous multi-attribute characteristics. Then it designed a heterogeneous multi-attribute decision-making model to calculate the relative closeness, and then used closeness to construct a value function to determine the weight index of each attribute. Finally, an empirical study was conducted on the urban agglomeration in Beijing-Tianjin-Hebei region of China. After years of environmental governance, the Beijing-Tianjin-Hebei region has gradually formed comprehensive environmental management measures against air pollution, water pollution, solid waste pollution and ecological environmental damage. Using the data of 13 cities from 2010 to 2018, the weight calculation and comprehensive index calculation of the environmental collaborative governance capability index of China's BeijingTianjin-Hebei regional urban agglomeration were carried out. The evaluation methods and results provide a scientific basis for the selection of comprehensive governance measures for cross-regional environmental collaborative governance.
\end{abstract}

Keywords: air pollution; cross-region; collaborative governance; evaluation index; multi-attribute decision-making

\section{Introduction}

Environmental governance is an increasingly serious global problem. In the past, many studies on environmental governance were based on urban environmental management. However, it is worth noting that air pollution, water pollution and other problems in environmental issues are all regional issues. The pollution sources in a certain area and the surrounding areas are in the same air flow field or in the same waters. Therefore, the pollutants of multiple cities affect each other. The environmental quality of this area is not only affected by other cities, but also directly or indirectly affects other cities. Therefore, the regional coordinated governance of environmental issues can be said to be both prosperous and 
all damaged, and it is difficult for a single region or city to take care of themselves.

The Beijing-Tianjin-Hebei region of China is one of the most heavily polluted areas in China. Through comprehensive regional governance, air and water pollution in the Beijing-Tianjin-Hebei region can be effectively alleviated. The number of heavily polluted days in Beijing, Tianjin and Hebei province was reduced to 15 days, 10 days, and 17 days respectively, and the air quality reached the best level in recent years. In terms of water pollution control, a cross-regional horizontal ecological compensation mechanism has been established for water diversion from Luanhe River to Tianjin and Chaobai River in the upper reaches of Miyun Reservoir to promote the comprehensive management of Yongding River, Baiyangdian Lake and Hengshui Lake.

\section{Method}

\subsection{Index system design}

The cross-regional environmental collaborative governance capability evaluation index system can be defined as a series of inter-related, which can dynamically and accurately reflect the cross-regional environmental collaborative governance status and the organic composition of the indicators. It contains 6 first-level indicators for population, environment, resources, economy, transportation, science and education investment, and 25 second-level indicators. The degree of ecological sustainable development takes into account ecological pressure, ecological overload, resource utilization and moderate population, so six indicators are set: green coverage rate in built-up areas (\%), total population (10,000 people), land utilization rate (\%), unit GDP SO2 emissions ( $\mathrm{Kg} / 10,000$ yuan), waste water emissions per unit GDP ( Kg/10,000 yuan) and efficient utilization of energy resources; the integration of high-quality development of the green economy is mainly considered, so 6 indicators are set: environmental protection patent application Percentage of data (\%), proportion of technology expenditure (\%), per capita GDP (yuan), per capita disposable income of urban residents (yuan), investment in environmental pollution control (100 million yuan), per capita retail sales of consumer goods (100 million yuan). The degree of green livability mainly considers the medical environment, education environment, transportation environment and natural environment, so six indicators are set: the rate of good ambient air quality (\%), the number of doctors per 10,000 people (persons), and the number of college students per 10,000 people (Person), harmless treatment rate of domestic garbage (\%), urban road area per capita (square meters) and recycling rate of renewable resources.

\subsection{Heterogeneous multi-attribute characteristics of indicators}

The indicators involved in this indicator system include quantitative indicators and qualitative indicators. Quantitative indicators are usually expressed in real numbers, but this article studies the long-term dimension, so such indicators are expressed in interval numbers. For qualitative indicators, resource utilization is characterized by certain fluctuations over time, so triangular fuzzy numbers should be used to express. When evaluating policy implementation, experts will give three evaluations: good, fair, and poor, so intuitive fuzzy numbers are used, which is more appropriate. Therefore, the indicators in this article mainly include three categories: interval numbers, triangular fuzzy numbers and intuitionistic fuzzy numbers.

2.3 Importance index of heterogeneous decision problems

Multi-attribute decision-making is to sort or optimize a limited number of options based on comprehensive consideration of multiple attributes. Because decision-making has a wide practical background in the field of social management, the research on decision-making theory and methods has always been a very important research direction in the field of management science and systems engineering.

Evaluation is a multi-attribute decision-making problem. How can the heterogeneous and multi-attribute ecological green integration indicators be specifically quantified in the form of importance index? This article analyzes the general 
definition of importance index in heterogeneous multi-attribute decision-making, and proposes a calculation method for importance indicators in heterogeneous multi-attribute decision-making.

\subsection{Heterogeneous multi-attribute decision-making model based on closeness}

In a multi-attribute decision-making problem, the preference information of a given decision maker is a positive ideal solution for each attribute value of the decision plan. The closeness indicates how close the attribute values are to each other. The higher the closeness of the attribute value to the positive ideal solution, the greater the contribution of the attribute. Therefore, the importance of attributes can be defined by relative closeness, so that the importance index can simultaneously reflect the consistency of subjective and objective requirements and changing trends.

\subsubsection{Normalization of heterogeneous information}

Since the dimension of each attribute value is different, it is necessary to standardize each attribute value. According to the benefit attribute and cost attribute, the normalization methods are as follows, take $e_{i j}$ as an example. The normalization of interval number $\mathrm{e}_{\mathrm{ij}}=\left[\mathrm{e}_{\mathrm{ij}}, \overline{\mathrm{e}}_{\mathrm{ij}}\right]\left(\mathrm{j} \in \mathrm{S}_{1}\right)$ is shown in formula (1).

$$
x_{i j}=\left\{\begin{array}{c}
{\left[\mathrm{e}_{i j} / \mathrm{e}_{\max }^{-}, \bar{e}_{i j} / \mathrm{e}_{\max }^{-}\right], j \in \mathrm{S}_{1}^{\mathrm{b}}} \\
{\left[1-\mathrm{e}_{\mathrm{ij}} / \mathrm{e}_{\max }^{-}, 1-\overline{\mathrm{e}}_{\mathrm{ij}} / \mathrm{e}_{\max }^{-}\right], \mathrm{j} \in \mathrm{S}_{1}^{\mathrm{c}}}
\end{array}\right.
$$

In the formula $\mathrm{e}_{\max }^{-}=\max \left\{\bar{e}_{\mathrm{ij}} \mid \mathrm{i}=1,2, \ldots, \mathrm{m}\right\}$.

The normalization of the triangular fuzzy number $\mathrm{e}_{\mathrm{ij}}=\left(\mathrm{a}_{\mathrm{ij}}, \mathrm{b}_{\mathrm{ij}}, \mathrm{c}_{\mathrm{ij}}\right)\left(\mathrm{j} \in \mathrm{S}_{2}\right)$ is shown in equation (2)

$$
x_{i j}=\left\{\begin{array}{l}
{\left[\frac{a_{i j}}{c_{\operatorname{maxj}}}, \frac{b_{i j}}{c_{\text {maxj }}}, \frac{c_{i j}}{c_{\text {max }}}\right], j \in S_{2}^{b}} \\
{\left[\frac{1-a_{i j}}{c_{\text {maxj }}}, \frac{1-b_{\text {ij }}}{c_{\text {maxj }}}, \frac{1-c_{i j}}{c_{\text {maxj }}}\right], j \in S_{2}^{c}}
\end{array}\right.
$$

In the formula $c_{\operatorname{maxj}}=\max \left\{c_{\mathrm{ij}} \mid \mathrm{i}=1,2, \ldots, \mathrm{m}\right\}$.

The intuitionistic fuzzy number $\mathrm{e}_{\mathrm{ij}}=\left\langle\mu_{\mathrm{ij}}, \mathrm{v}_{\mathrm{ij}}\right\rangle\left(\mathrm{j} \in \mathrm{S}_{3}\right)$ is between 0 and 1 , without normalization.

\subsubsection{Relative closeness of heterogeneous information}

In heterogeneous multi-attribute decision-making, closeness is usually used to reflect the closeness of decisionmaking schemes. In order to compare the schemes, calculate the positive and negative ideal solutions of heterogeneous information, as two reference points, denoted as $\mathrm{x}^{+}$and $\mathrm{x}^{-}, \mathrm{x}^{+}=\left(\mathrm{x}_{1}^{+}, \mathrm{x}_{2}^{+}, \ldots, \mathrm{x}_{\mathrm{n}}^{+}\right), \mathrm{x}^{-}=\left(\mathrm{x}_{1}^{-}, \mathrm{x}_{2}^{-}, \ldots, \mathrm{x}_{\mathrm{n}}^{-}\right)$. The positive ideal solution refers to the most satisfactory solution for each attribute, and the negative ideal solution refers to the most unsatisfactory solution for each attribute.

$$
x_{j}^{+}=\left\{\begin{array}{c}
{\left[e_{j}^{+}, e_{j}^{\mp}\right], j \in S_{1}} \\
\left(a_{j}^{+}, b_{j}^{+}, c_{j}^{+}\right), j \in S_{2} \\
\left\langle\mu_{j}^{+}, v_{j}^{+}\right\rangle, j \in S_{3}
\end{array}\right.
$$

In the formula $e_{i}^{+}=\max _{\substack{i=1,2, \ldots, m \\ j=1,2, \ldots, n}}\left\{e_{i j}\right\}, \bar{e}_{i}^{+}=\max _{\substack{i=1,2, \ldots, m \\ j=1,2, \ldots, n}}\left\{\bar{e}_{i j}\right\}, a_{i}^{+}=\max _{\substack{i=1,2, \ldots, m \\ j=1,2, \ldots, n}}\left\{a_{i j}\right\}, b_{i}^{+}=\max _{\substack{i=1,2, \ldots, m \\ j=1,2, \ldots, n}}\left\{b_{i j}\right\}, c_{i}^{+}=\max _{\substack{i=1,2, \ldots, m \\ j=1,2, \ldots, n}}\left\{c_{i j}\right\}, v_{i}^{+}=$ $\max _{\substack{i=1,2, \ldots, m \\ j=1,2, \ldots, n}}\left\{v_{i j}\right\}$. 
Similarly,

$$
x_{j}^{-}=\left\{\begin{array}{c}
{\left[e_{j}^{-}, \bar{e}_{j}^{-}\right], j \in S_{1}} \\
\left(a_{j}^{-}, b_{j}^{-}, c_{j}^{-}\right), j \in S_{2} \\
\left\langle\mu_{j}^{-}, v_{j}^{-}\right\rangle, j \in S_{3}
\end{array}\right.
$$

Suppose that the relative closeness between the decision maker's decision preference and the positive ideal solution of heterogeneous information is

$$
z_{i j}=\frac{d\left(x_{i j}, x^{+}\right)}{d\left(x_{i j}, x^{+}\right)+d\left(x_{i j}, x^{-}\right)}
$$

In the formula,

$$
\begin{gathered}
d\left(x_{i j}, x^{+}\right)=\left\{\begin{array}{c}
\frac{1}{2}\left[\left(d_{i j}-e_{i}^{+}\right)^{2}+\left(d_{i j}-\bar{e}_{i}^{+}\right)^{2}\right], j \in S_{1} \\
\frac{1}{3}\left[\left(a_{i j}-a_{i}^{+}\right)^{2}+\left(b_{i j}-b_{i}^{+}\right)^{2}+\left(c_{i j}-c_{i j}^{+}\right)^{2}\right], j \in S_{2} \\
\frac{1}{L^{2}}\left(\Delta^{-1}\left(x_{i j}, 0\right)-\Delta^{-1}\left(x^{+}, 0\right)\right)^{2}, j \in S_{3} \\
\frac{1}{3}\left[\left(\mu_{i j}-\mu_{i}^{+}\right)^{2}+\left(v_{i j}-v_{i}^{+}\right)^{2}+\left(\pi_{i j}-\pi_{i}^{+}\right)^{2}\right], j \in S_{4}
\end{array}\right. \\
d\left(x_{i j}, x^{-}\right)=\left\{\begin{array}{c}
\frac{1}{2}\left[\left(d_{i j}-e_{i}^{-}\right)^{2}+\left(d_{i j}-\bar{e}_{i}^{-}\right)^{2}\right], j \in S_{1} \\
\frac{1}{3}\left[\left(a_{i j}-a_{i}^{-}\right)^{2}+\left(b_{i j}-b_{i}^{-}\right)^{2}+\left(c_{i j}-c_{i}^{-}\right)^{2}\right], j \in S_{2} \\
\frac{1}{L^{2}}\left(\Delta^{-1}\left(x_{i j}, 0\right)-\Delta^{-1}\left(x^{-}, 0\right)\right)^{2}, j \in S_{3} \\
\frac{1}{3}\left[\left(\mu_{i j}-\mu_{i}^{-}\right)^{2}+\left(v_{i j}-v_{i}^{-}\right)^{2}+\left(\pi_{i j}-\pi_{i}^{-}\right)^{2}\right], j \in S_{4}
\end{array}\right.
\end{gathered}
$$

\subsubsection{Heterogeneous multi-attribute model calculation}

Since the above relative closeness reflects the closeness of the attribute value to the positive ideal solution, its value can be approximated as the contribution of the attribute. The smaller the $z_{i j}$ value, the higher the closeness and the greater the contribution; the larger the $z_{i j}$ value, the lower the closeness and the smaller the contribution. The relative closeness is further processed to make the value larger and the affinity higher, which is used as the utility function $v$ to calculate the importance index of heterogeneous multi-attribute decision-making. Let $z^{\prime}=1-z$ the calculation of the heterogeneous multi-attribute model based on closeness is shown in equation (8).

$$
\emptyset_{\mathrm{j}}\left(\mathrm{z}^{\prime}\right)=\sum_{\mathrm{i}=1}^{\mathrm{m}} \frac{\left(\mathrm{n}-\sigma\left(\mathrm{S}_{-1}\right)-1\right) ! \mathrm{k}\left(\mathrm{S}_{-\mathrm{i}}\right) !}{\left(\mathrm{n}+\mathrm{k}\left(\mathrm{S}_{-\mathrm{i}}\right)-\sigma\left(\mathrm{S}_{-\mathrm{i}}\right)\right) !}\left(\mathrm{z}_{\mathrm{i}}^{\prime}\left(\mathrm{S}_{-\mathrm{j}}, \mathrm{k}_{\mathrm{j}}\right)-\mathrm{z}_{\mathrm{i}}^{\prime}\left(\mathrm{S}_{-\mathrm{j}}, 0_{\mathrm{j}}\right)\right), \forall \mathrm{j} \in \mathrm{N}
$$

After obtaining the multi-attribute decision weights based on closeness, the cross-regional environmental collaborative governance capability index is measured according to the linear fitting formula as shown in equation (9).

$$
\mathrm{P}_{\mathrm{it}}=\sum_{\mathrm{j}=1}^{\mathrm{n}} \emptyset_{\mathrm{j}}\left(\mathrm{z}^{\prime}\right) \times \mathrm{x}_{\mathrm{ij}}, \mathrm{i}=1,2, \ldots, 27 ; \mathrm{j}=1,2, \ldots, 20 ; \mathrm{t}=2010,2011, \ldots, 2018
$$

\section{Empirical Analysis and Result Discussions}

3.1 Research scope and data sources

China's Beijing-Tianjin-Hebei urban agglomeration has an important strategic position at the national level. At the end of 2018, the total population of the Beijing-Tianjin-Hebei region reached 11.701 million, accounting for $8.1 \%$ of the country's total population. The total GDP reached 3249.46 billion yuan, accounting for $8.6 \%$ of the country's total GDP. At the same time, the Beijing-Tianjin-Hebei urban agglomeration is also the most concentrated and severe area of smog pollution in China. The environmental problems of China's Beijing-Tianjin-Hebei region have become a difficult problem in the implementation of the regional coordinated development strategy. In order to realize the potential of the above- 
mentioned comprehensive environmental management measures, the comprehensive environmental management measures in the Beijing-Tianjin-Hebei region have been systematically evaluated, which will provide environmental management departments with a refined comprehensive management decision-making basis. And it seems particularly important. The research scope of this paper covers 13 cities in the Beijing-Tianjin-Hebei region of China, including Beijing, Tianjin and 11 cities in Hebei Province (Shijiazhuang, Baoding, Tangshan, Qinhuangdao, Langfang, Xingtai, Chengde, Cangzhou, Hengshui, Handan, Zhangjiakou).

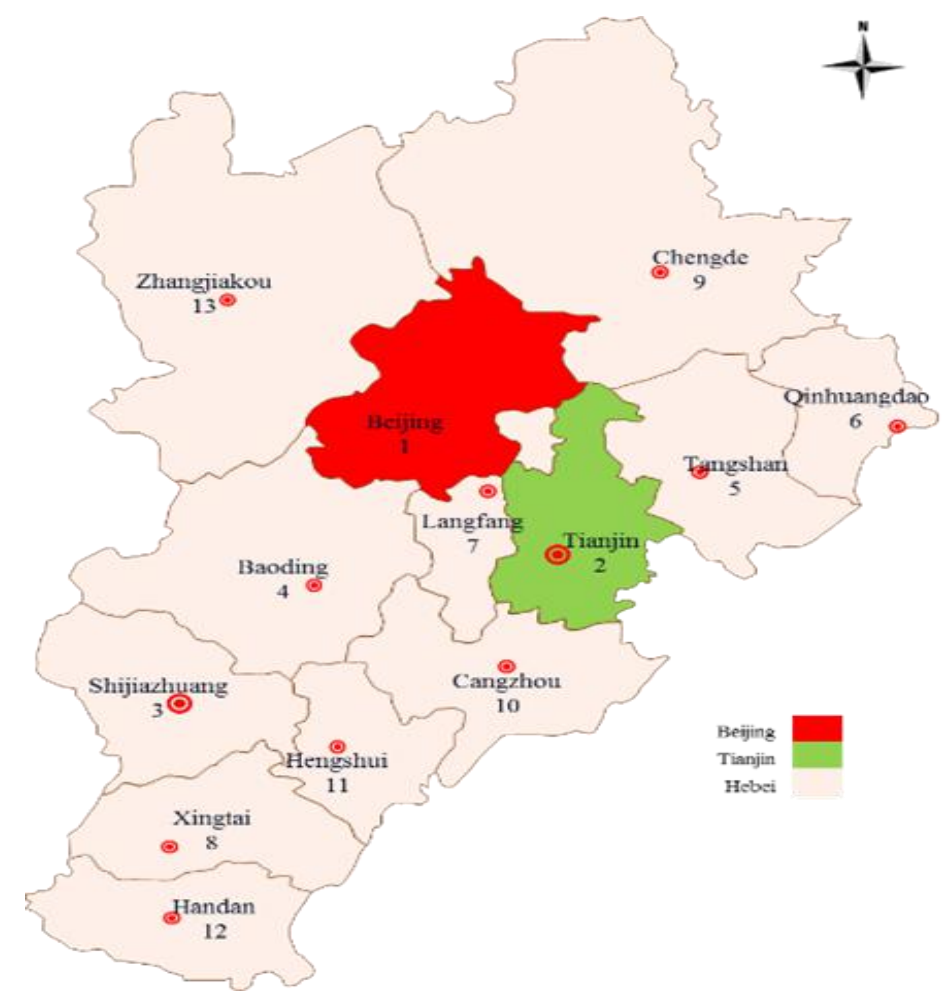

Figure 1. Location of the Beijing-Tianjin-Hebei region in China

In the calculations in this section, 13 cities in the Beijing-Tianjin-Hebei region are selected for analysis. Quantitative data are derived from the 2010-2018 China City Statistical Yearbook and the 13 cities' Statistical Yearbook and National Economic and Social Development Statistical Bulletin, "Economic Yearbook", "Economic Statistics Yearbook", etc., some of the environmental data comes from the Beijing-Tianjin-Hebei sub-industry GDP and per capita GDP (2005-2018), the situation of Beijing-Tianjin-Hebei medical and health institutions (2005 -2018), Beijing-Tianjin-Hebei social security situation (2005-2018), Beijing-Tianjin-Hebei population situation (2005-2018), etc. Some missing data in the historical data are processed by interpolation. Qualitative indicators come from expert evaluation.

\subsection{Index calculation and result analysis}

First, evaluate the cross-regional environmental governance capabilities of 13 cities in the Beijing-Tianjin-Hebei region of China. Determine the evaluation value of each indicator in each plan through expert opinions, language variables and statistical methods. Then, standardize and calculate the heterogeneous information for the positive and negative ideal solutions of heterogeneous information, the relative closeness is obtained, and the weight of each index is calculated based on the heterogeneous multi-attribute model of closeness. Finally, the weighted average method is used to obtain the 20102018 China Beijing-Tianjin-Hebei urban agglomeration environmental collaborative governance capability index.

\section{Conclusions}

This paper firstly analyzes the development history of global cross-regional environmental governance. Combined with China's ecological civilization and regional ecological green development strategies, it gives a multi-attribute cross- 
regional environmental collaborative governance capability evaluation index system. And then it designs a heterogeneous multi-variety based on closeness. The attribute decision-making model, combined with the data of 13 cities in the BeijingTianjin-Hebei urban agglomeration from 2010 to 2018, conducts an empirical analysis to obtain the weight values of related indicators, and then uses the weighted average method to obtain the comprehensive index. In general, China's comprehensive cross-regional environmental management measures can be summarized into five categories: industrial structure adjustment, energy structure adjustment, transportation structure adjustment, land use structure adjustment, and agricultural and rural green transformation. As China's Beijing-Tianjin-Hebei comprehensive environmental management is difficult, it faces the challenge of comprehensive management of air, water, and solid waste pollution and the improvement of the ecological environment. The sources of pollution are complex, including point source pollution like industrial enterprises, line source pollution from road traffic and non-point source pollution from agriculture and rural areas. After years of environmental management, the Beijing-Tianjin-Hebei region has gradually formed five types of comprehensive environmental management measures: industrial structure adjustment, energy structure adjustment, transportation structure adjustment, land use structure adjustment, and agricultural and rural green transformation. Industrial structure adjustment measures: efforts will be made to improve the level and capabilities of regional industrial intelligent manufacturing and develop mid-to-high-end service industries. Energy structure adjustment measures: implement coal total control, increase the proportion of natural gas consumption, and strive to develop new energy sources. Traffic and transportation structure adjustment measures: improve and increase the combined transportation capacity of railways and ships, and implement high-standard cars or electric vehicles in urban passenger transportation. Land use structure adjustment measures: strictly control construction land, and continue to implement land hardening and greening projects. Agricultural and rural green transformation measures: appropriate use of pollutant treatment methods according to local conditions, precise fertilization and fertilizer saving, control of pesticide application, and implementation of rural natural gas transformation.

\section{Conflicts of Interest}

The author declares no conflicts of interest regarding the publication of this paper.

\section{References}

[1] Gulia S., Nagendra S.M.S., Barnes J. and Khare M. (2018). Urban Local Air Quality Management Framework for Non-attainment Areas in Indian Cities. Science of The Total Environment, 619-620 (220):1308-1318.

[2] The Paper News. (2019). Beijing-Tianjin-Hebei "Change in Breathing" Reflects the Effect of Ecological Civilization Construction, http://www.xinhuanet.com/energy/2019-02/28/c 1124173502.htm.

[3] Michel G.,Christophe L. and Mustapha R. (2019). On Importance Indices in Multicriteria Decision Making. European Journal of Operational Research, 277(1):269-283.

[4] Yu G.F., Li D.F., Ye Y.F and Qiu J.M. Heterogeneous Multi-attribute Variable Weight Decision Making Method Considering Regret Aversion. Computer Integrated Manufacturing Systems, 23(01):154-161.

[5] Beijing Municipal Bureau Statistics. (2020). Survey Office of the National Bureau of Statistics in Beijing, http://tjj.beijing.gov.cn/. 